University of Nebraska - Lincoln

DigitalCommons@University of Nebraska - Lincoln

Faculty Publications in Computer \& Electronics Electrical \& Computer Engineering, Department Engineering (to 2015)

2008

\title{
Truncated Hyperbolic Mapping for Non-binary Turbo Coding in UWB
}

\author{
K. Popovski \\ University of Wollongong \\ Tadeusz Wysocki \\ University of Nebraska-Lincoln, wysocki@uow.edu.au \\ B. J. Wysocki \\ University of Nebraska-Lincoln
}

Follow this and additional works at: https://digitalcommons.unl.edu/computerelectronicfacpub

Part of the Computer Engineering Commons

Popovski, K.; Wysocki, Tadeusz; and Wysocki, B. J., "Truncated Hyperbolic Mapping for Non-binary Turbo Coding in UWB" (2008). Faculty Publications in Computer \& Electronics Engineering (to 2015). 26. https://digitalcommons.unl.edu/computerelectronicfacpub/26

This Article is brought to you for free and open access by the Electrical \& Computer Engineering, Department of at DigitalCommons@University of Nebraska - Lincoln. It has been accepted for inclusion in Faculty Publications in Computer \& Electronics Engineering (to 2015) by an authorized administrator of DigitalCommons@University of Nebraska - Lincoln. 


\title{
Truncated Hyperbolic Mapping for Non-binary Turbo Coding in UWB
}

\author{
K. Popovski ${ }^{\dagger}$, T. A. Wysocki ${ }^{\dagger \dagger}$, Senior Member, IEEE, and B. J. Wysocki ${ }^{\dagger}$ \\ ${ }^{\dagger}$ School of Electrical, Computer and Telecommunications Engineering \\ University of Wollongong, Northfields Ave, Wollongong, NSW, 2522, Australia \\ Email: $\{$ keni, bjw\}@uow.edu.au \\ ${ }^{\dagger \dagger}$ Department of Computer and Electronics Engineering \\ University of Nebraska - Lincoln, 1110 South 67 St, Omaha, NE, 68182-0572, United States of America \\ Email: twysocki@mail.unomaha.edu
}

\begin{abstract}
The combination of user multiplexing and data encoding within Ultra Wideband systems has been proposed to improve bit error performance. Relative to conventional binary coding, this technique has shown to reduce error rates in scarcely populated systems. Where non-binary turbo coding modulates data to form an integer sequence, a random mapping is generally applied to develop a time hopping sequence. This paper explores the possibility of mapping through the application of truncated hyperbolic congruence codes. Comparative results against a random mapping indicate an improvement in system performance for higher turbo iteration levels in the receiver. Transceiver architecture is based upon the transmitter side equalization approach of time reversed communications.
\end{abstract}

Index Terms-UWB, time-reversed, pre-rake equalization, TCM, TTCM, nonbinary-turbo, hyperbolic congruence

\section{INTRODUCTION}

$\mathbf{U}$ LTRA Wideband (UWB) has seen increased attention since its release for commercial applications in early 2002 [1]. It is characterized by having a fractional bandwidth of more than $20 \%$, or bandwidth occupancy greater than 500 $\mathrm{MHz}$ [2]. Although several techniques exist for UWB communications, this paper is focused on time hopped time reversed UWB (TH-TR-UWB) [3]. This time reversed scheme has also been referred to as 'pre-rake' [4]. While a conventional system would operate with the transmission of sub-nanosecond width Gaussian waveforms, a time reversed system uses the channel impulse response from the transmitter to the receiver as a transmit pre-filter.

Within traditional time hopped UWB, pulses transmitted are either delayed in time (pulse position modulation (PPM)) or changed in amplitude (pulse amplitude modulation (PAM)) for encoding data. TH-UWB systems generally use code division multiple access based upon a family of orthogonal time hopping codes for the multiplexing of users.

Turbo coding may also be applied in order to attain a performance closer to the capacity of the communication channel. This paper applies the technique of combining data encoding with time hopping, achieved through non-binary turbo codes, introduced in [5]. This encodes information symbols onto an expanded modulation set, rather than binary pulse encoding combined with a preset time hopping code. Trellis turbo coded modulation (TTCM) is utilized, which was first presented by Robertson and Worz [6]. However, with the increased performance comes an inherent increase in system complexity.

TTCM uses a parallel concatenation of two binary trellis coded modulation (TCM) encoders. TCM optimizes the Euclidean distance between codewords by combining rate $R=m /(m+1)$ binary convolutional codes with an $M$-ary signal constellation [7]. Coding gains are achievable without a subsequent bandwidth expansion by replacing the component codes in a binary encoder with trellis codes [8]. Increased performance is possible relative to classical binary coding, with TTCM having a better convergence of the iterative decoding, less sensitivity to puncturing patterns, and greater robustness toward the flaws of the decoding algorithm [9]. Decoding within a TTCM system is conducted symbol by symbol, rather than bit by bit as in conventional turbo coding. A modified symbol based SOVA (soft output Viterbi algorithm) decoding procedure is applied within this paper, based upon maximum likelihood accumulated metrics through the trellis.

An additional degree of user differentiation is achieved through a random PPM shifting.

The link between the TTCM constellation $\left(\left[0,2^{m+1}-1\right]\right)$ and the time hopping integer range is achieved through a unique memoryless mapper. This generates a one-to-one correspondence between the coded bits and the time hopping chip positions. While a random hopping sequence is generally used, this paper explores the application of hyperbolic congruence codes for this mapping. Random and hyperbolic codes have been shown to achieve a similar BER performance in a turbo coded system for a high interleaver size [10]. Application herein, however, is through truncated codes shorter than the sequence periodicity.

This paper is organized as follows: Section II presents an overview of the UWB system architecture studied, Section III discusses TTCM using both random and hyperbolic mapping, and comparative results are shown in Section IV. Concluding statements are given in Section V.

\section{UWB ARCHITECTURE}

The signal $x^{(u)}(t)$ transmitted for the $u$ th user in a timehopped time-reversed UWB system requires pre-filtered using 
the time-reversed complex conjugate of the forward link channel response. Having equiprobable data $b_{j}^{(u)} \in\{-1,1\}$ mapped through binary PPM, and time shift $\varepsilon$ set to equal the pulse width, the transmitted signal is defined as:

$$
\begin{array}{r}
x^{(u)}(t)=\frac{\sqrt{E_{T X}(u)}}{\sqrt{G_{H, u ; x_{p o s}}}}\left(\sum_{j=0}^{n-1} w\left(t-j T_{f}-c_{j}^{(u)} T_{c}-\varepsilon b_{j}^{(u)}\right)\right) \\
\otimes h\left(u ; x_{p o s},-t\right),
\end{array}
$$

where $\otimes$ represents convolution, $E_{T X}(u)$ is the user signal energy, $G_{H, u ; x_{\text {pos }}}$ represents the gain of the channel required for normalization, $w(t)$ is the base transmitted waveform of width $T_{w}$ seconds, $j$ is the frame number, and $x_{\text {pos }}$ represents the position of the receiver. $T_{f}$ is a single frame length, which is segmented into $N_{h}$ equally spaced intervals called 'chips' of duration $T_{c}$, such that $T_{f}=N_{h} T_{c} . c_{j}^{(u)}$ denotes the position within the particular frame (the chip number) that is occupied by the $u$ th user's signal in accordance with a time hopping sequence. A perfectly power controlled system is studied, whereby $E_{T X}$ is constant for all users.

The signal received, taking to account contributions of all $N_{u}$ users, is given by:

$$
y(t)=\left(\sum_{u=1}^{N_{u}} x^{(u)}(t) \otimes h\left(u ; x_{p o s}, t\right)\right)+n(t)
$$

All transmitters were assumed dispersed enough such that the channel responses from each $N_{u}$ transmitter to any receiver are independent. As such, each convolution is calculated using the response from user $u$ to the desired receiver. The final parameter in this function is additive white Gaussian noise with variance $\sigma^{2}=N_{0} / 2$. The signal to noise ratio is defined as a function of the received signal energy and the noise variance as $\operatorname{SINR}=E_{R X}(u) / \sigma^{2}$, where $E_{R X}(u)$ is the received signal energy per symbol.

The base pulse $w(t)$ was set as the second derivative of the Gaussian pulse, with center frequency $f_{0}$, defined as [11]:

$$
w(t)=\left[1-2\left(\pi t f_{0}\right)^{2}\right] \exp \left\{-\left(\pi t f_{0}\right)^{2}\right\} .
$$

A center frequency of $3.9 \mathrm{GHz}$ was used, which results in a monocycle width of $T_{w}=0.5 \mathrm{~ns}$.

A chip synchronous single-input-single-output (SISO) system is considered within this paper. Transmit and receive antennas, which would act as pulse shaping filters, are assumed to have no significant effect on the signal transmitted. Time reversal properties still apply in a SISO system, assuming that the bandwidth occupied by transmissions is much larger than the correlation frequency exhibited by the channel [12].

The multipath model applied is the IEEE 802.15.3a channel, based on the SV (Saleh-Valenzuela) model where multipath components arrive in clusters [13]. The LOS channel scenario (CM1) was selected for all testing. A quasi-stationary model is assumed, remaining time-invariant for the transmission of a block of data, and independent between blocks.

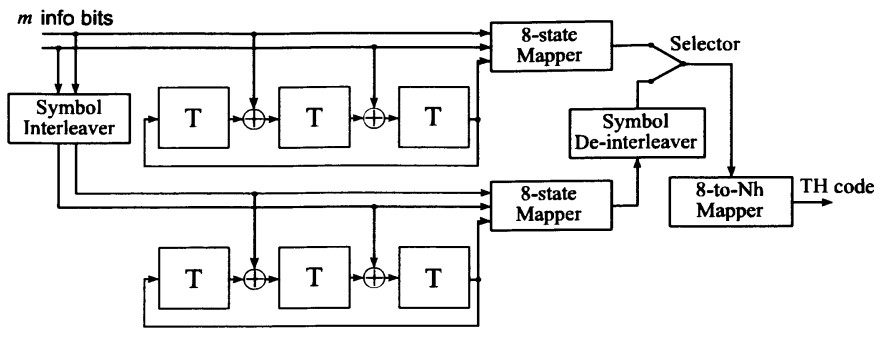

Fig. 1. TTCM transmitter structure

\section{TTCM SYSTEM INTEGRATION}

\section{A. TTCM Transmitter}

The incorporation of a TTCM transmitter into a UWB system allows the combination of the time hopping stage with the data encoding state. This entails the determination of the time hopping sequence $c_{j}^{(u)}$. Using $m=2$ data bits, an 8 constellation point output is generated. In contrast, a conventional binary turbo coding architecture operates at the binary data level, altering $b_{j}^{(u)}$ in accordance with a preset convolutional code.

The structure of a TTCM transmitter is illustrated in Fig. 1. The parallel concatenation of two identical $m$-ary recursive systematic convolutional (RSC) encoders here operates on $m$ bit-words. The $2^{m}$ possible inputs are encoded using a code rate of $m / m+1$, with the encoded $m+1$ bits mapped through the function $\bar{\Pi}(j)$ to one of $2^{(m+1)}$ constellation points [8], [14]. This $m+1$ bit output, with $m=2$, consists of two systematic bits, together with a single parity bit. The encoder selects the symbols alternately from the two TCM encoders, resulting in an aligned systematic component between the encoders, with only the parity bit being alternately chosen. A total of $\gamma=m+1$ shift registers are utilized in each RSC encoder.

The interleaver within this TTCM transmitter must operate on a pairwise basis, where even blocks of $m$ bits are mapped to even positions, and odd blocks of $m$ bits mapped to odd positions [6], [8], [14]. This symbol interleaver is applied on the data input before processing with the lower RSC encoder, with a de-interleaving operation conducted on the result to ensure the ordering of the systematic component between both encoders. Constraints imposed on the component code include that no parallel transitions should exist in the corresponding trellis diagram of the convolutional encoder. This is to ensure each data bit benefits from the interleaving and parallel concatenation. Also, the information bits in an arbitrary step $k$ should not directly influence the parity bit at step $k[6]$.

It can be noted in this TTCM transmitter that trellis termination is not applied, which will inevitably lead to a slight performance degradation relative to a turbo system with termination capabilities. Also, unlike in conventional turbo, this transmitter always applies symbol puncturing. A uniform probability of the occurrence of each constellation point may be verified through a histogram based analysis of this punctured output, considering a random interleaver design with a Bernoulli mix input binary stream. 
Since simulations conducted in this paper are chip synchronous, a random PPM shift $(\varepsilon)$ must be applied in order to diversify the TTCM signals further. This scattering is done based upon the aforementioned 8 point mapper. With $m=2$ bits transmitted per $N_{s}$ pulses (all $N_{s}$ pulses having the same chip position), the number of pulses required for data transmission is effectively halved. For comparable results, the energy of each pulse in this TTCM system is doubled.

The final block in this TTCM structure $\left(8-t o-N_{h}\right.$ Mapper) maps the 8 possible constellation points through $\bar{\Pi}(j)$ uniquely onto the $N_{h}$ possible chip positions utilized for UWB time hopping. A random mapping function is common, although does not provide the advantages of deterministic codes. These include reduced complexity of design, as hyperbolic codes may be stored within a look-up table. Various multiuser sequencing methods may be adopted, provided unique elements are generated. Although the sequence ordering is not sustained, which is vital in obtaining code orthogonality, truncated structured codes will on average obtain a more effective use of the $N_{h}-8$ unused time slots over all users. Where a random mapping will uniformly select positions from the $N_{h}$ possible time chips, a properly designed code family will seek a uniform chip use over all users. This subsequently leads to a uniform selection of the unused slots, thus achieving a better system performance. This paper adopts hyperbolic congruence codes for comparison with randomly selected sequences. Noting $u$ as the user number $\left(0<u \leq N_{h}\right)$, and taking $p$ to be a prime integer, hyperbolic codes are designed through the placement operator [15]:

$$
\bar{y}(j)=u / j(\bmod p) .
$$

This family of codes allows a maximum of $p-1$ users, with a cardinality of $p-1$ and a periodicity of $p$. Within prime number based hopping methods, the code cardinality $N_{h}$ is set by the parameter $p$.

\section{B. TTCM Receiver}

1) Decoder Structure: The TTCM receiver is similar in structure to that for conventional binary turbo codes, both based upon an iterative process. However, within a non-binary system sets of log-likelihood ratios (LLRs) are passed between the component decoders, rather than single log-likelihoods [16]. Each iteration seeks to improve the log-likelihood ratio, converging to an estimate of the transmitted codeword. The underlying SOVA algorithm in a TTCM system is also more complex, as the systematic and extrinsic components cannot be separated since noise simultaneously affects the parity and systematic bits [6]. Here the output is split into two components: the a priori information $\left(L_{a}\right)$ and the combined extrinsic and systematic information $\left(L_{e \& s}\right)$, with the latter being interleaved/de-interleaved and passed between alternate decoders. A decision is conducted on the final LLRs to evaluate the symbol that was received.

The receiver structure and symbol-based SOVA algorithm with maximum sequence posteriori probability estimations discussed herein is adapted from [14] and [17]. The structure used is shown in Fig. 2.

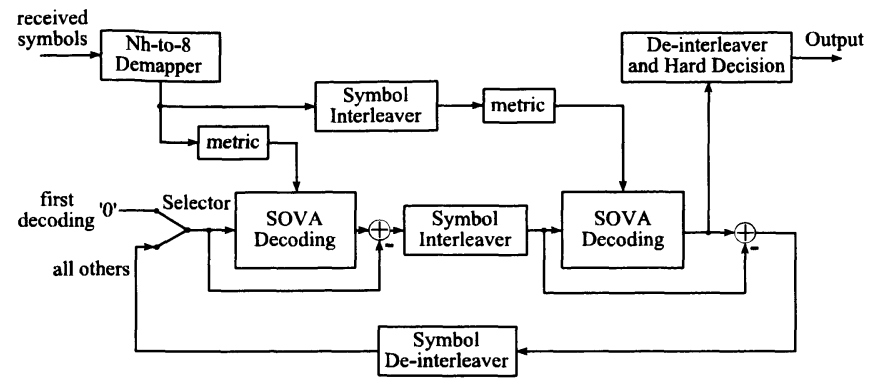

Fig. 2. TTCM receiver structure

The received signal in each of the $N_{h}$ chips is correlated with a preset template of the expected received signal, and passed to the receiver as soft data. Collection of correlation values is conducted over all $N_{s}$ transmitted signals. The $2^{m}$ possible transmission locations are then de-mapped through $\bar{\Pi}^{-1}(j)$. The $2^{m}$ a priori values are given a zero neutral probability on the first iteration, since the transmitter utilized puncturing between the component encoders.

2) Symbol Based SOVA: The component encoder state can be represented as $S_{k} \in\left\{0,1, \ldots, 2^{\gamma}-1\right\}$, with $\gamma$ shift registers in each RSC encoder. The $m$ bit input is denoted as $d_{k} \in\left\{0,1, \ldots, 2^{m}-1\right\}$, with received sequence $y_{1 \rightarrow N}=$ $\left(y_{1}, y_{2}, \ldots, y_{N}\right)$ shown from time 1 to $N$. The LLR value for each possible symbol $i$ at time $k$ is calculated as:

$$
L\left(d_{k}=i\right) \triangleq \log \frac{\operatorname{Pr}\left(d_{k}=i \mid y_{1 \rightarrow N}\right)}{\operatorname{Pr}\left(d_{k}=0 \mid y_{1 \rightarrow N}\right)},
$$

with the a priori information being:

$$
L_{a}\left(d_{k}=i\right)=\log \frac{\operatorname{Pr}\left(d_{k}=i\right)}{\operatorname{Pr}\left(d_{k}=0\right)} .
$$

The cumulated path likelihood metric is represented as $M\left(\vec{S}_{k}^{s}\right)=\log \left(\operatorname{Pr}\left(\vec{S}_{k}^{s}, \vec{y}_{t \leq k}\right)\right)$, looking at time $k$ and current state $s$. The state vector is $\vec{S}_{k}^{s}$, which identifies the states along the survivor path terminated at state $S_{k}=s$.

The TTCM receiver trellis assumes that $2^{m}$ branches with distinct symbols enter each of the $2^{\gamma}$ trellis nodes. The parameter $q\left(d_{k}=i \mid S_{k-1}=s^{\prime}, S_{k}=s\right)$ is defined as the probability that symbol $i$ is associated with the transition from states $S_{k-1}=s^{\prime}$ to $S_{k}=s$. Based upon the metric equation for the binary SOVA algorithm, and applying the simplification procedure presented in [14], the cumulated path metric may be approximated as:

$$
\begin{aligned}
& M\left(\overrightarrow{S_{k}^{s}}\right)=M\left(\vec{S}_{k-1}^{s^{\prime}}\right)+ \\
& \quad L_{c} \cdot \log \left(p\left(y_{k} \mid d_{k}=i, S_{k}=s, S_{k-1}=s^{\prime}\right)\right) \\
& \quad+\left[L_{a}\left(d_{k}=i\right)-\max _{i \in\left(0,1, \ldots, 2^{m}-1\right)}\left(L_{a}\left(d_{k}=i\right)\right)\right],
\end{aligned}
$$

for $q\left(d_{k}=i \mid S_{k-1}=s^{\prime}, S_{k}=s\right)=1$, and:

$$
M\left(\vec{S}_{k}^{s}\right)=-\infty
$$

for $q\left(d_{k}=i \mid S_{k-1}=s^{\prime}, S_{k}=s\right)=0$. The term $\log \left(p\left(y_{k} \mid\right.\right.$ $\left.d_{k}=i, S_{k}=s, S_{k-1}=s^{\prime}\right)$ ) is calculated from the soft received values. The multiplication by the channel metric 
$L_{c}=4 a E_{T X} / 2 \sigma^{2}$ ensures that the reliance on received soft values is determined by the channel reliability relative to prior reliabilities.

The log likelihood ratio for each symbol is calculated through the maximum likelihood metric obtained through the Viterbi decoding on the $2^{m}$ paths entering each state. The parameter $\Gamma_{k, i}\left(\vec{S}_{N}^{s}\right)$ is defined as the reliability difference between the maximum likelihood symbol at time $k$ and the most likely codeword with $d_{k}=i$, both terminated at state $S_{N}^{s}$ :

$$
\Gamma_{k, i}\left(\vec{S}_{N}^{s}\right)=\max _{\text {all } \vec{S}_{N}^{s}}\left\{M\left(\vec{S}_{N}^{s}\right)\right\}-\max _{\vec{S}_{N}^{s}: d_{k}=i}\left\{M\left(\vec{S}_{N}^{s}\right)\right\} .
$$

The LLR values can be obtained as:

$$
L\left(d_{k}=i\right)=\Gamma_{k, 0}\left(\vec{S}_{N}^{s}\right)-\Gamma_{k, i}\left(\vec{S}_{N}^{s}\right) .
$$

The parameter $L\left(d_{k}=i\right)$ is estimated over $\delta=5 \gamma$ trellis increments in order to simplify calculations and limit the memory requirements at the receiver. Each state in the trellis stores a $\delta \times 2^{m}$ reliability measure matrix, under the assumption that the survivor and competition paths have converged over the $\delta$ time increments. The reliability difference between survivor and competing paths with $d_{k}=l$, and both terminated at state $S_{t+1}^{s}$, is denoted as:

$$
\Delta_{t+1, l}^{s}=\max _{\text {all } \vec{S}_{t+1}^{s}}\left(\vec{S}_{t+1}^{s}\right)-M\left(S_{t, l}, S_{t+1}^{s}\right),
$$

where $M\left(S_{t, l}, S_{t+1}^{s}\right)$ represents the cumulative path metric terminated at state $S_{t+1}^{s}$ and previous state $S_{t, l}$. It can be noted that $\Delta_{t+1, l}^{s}=0$ for the surviving path at state $S_{t+1}^{s}$. Initially, $\Gamma_{k, i}\left(\vec{S}_{k}^{s}\right)=\Delta_{k, i}^{s}$, with the remaining reliability values $\Gamma_{k, i}\left(\vec{S}_{t+1}^{s}\right)$ for $k=t-\delta+2, \ldots, k$ updated as [14], [17]:

$$
\Gamma_{k, i}\left(\vec{S}_{t+1}^{s}\right)=\min _{l=0,1, \ldots, 2^{m}-1}\left\{\Delta_{t+1, l}^{s}+\Gamma_{k, i}\left(\vec{S}_{t, l}\right)\right\} .
$$

The LLR value for each $d_{k}=i$ relative to $d_{k}=0$ is estimated over $\delta$ time positions in the trellis. It can be noted that $L\left(d_{k}=0\right)$ by definition will always give a neutral probability.

\section{Simulation Results}

A TR-UWB (time reversed UWB) simulation was adapted from a time hopped PPM UWB simulation designed by Di Benedetto and Giancola [18]. Perfect channel state information was assumed at the transmitter side. The cardinality and periodicity of each time hopping code were set to 11 , with a pulse width of $0.5 \mathrm{~ns}$, and a data encoding shift of $0.5 \mathrm{~ns}$. Transmit power was set to $1 \mathrm{~mW}$, with the results scalable. The pulse repetition parameter $N_{s}$ was set to 1 for all tests, with an data block length of 400 pulses. Random turbo interleavers were generated for each data block.

The performances of random and hyperbolic symbol mapping within a TTCM error correction system for a single user scenario are depicted in Fig. 3. A data rate of $50 \mathrm{Mbit} / \mathrm{s}$ was selected, with the error rate for a system not applying

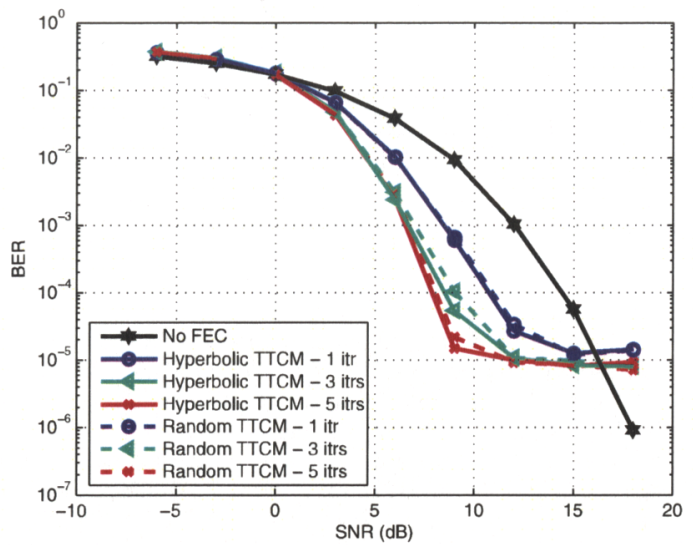

Fig. 3. Random vs hyperbolic symbol mapping in a single user scenario

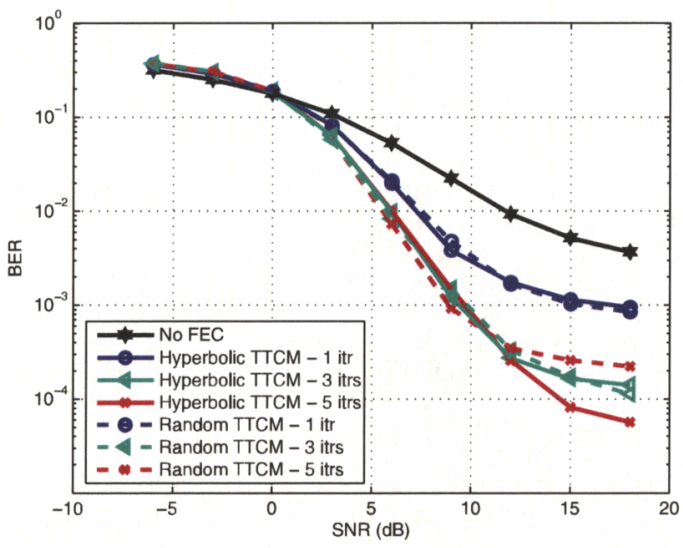

Fig. 4. Random vs hyperbolic symbol mapping in a multi user scenario

error correction shown for comparison. As the benefits of orthogonal sequencing only become apparent in a multi-user scenario, random and the hyperbolic mapping achieve similar error performance levels. An error floor for both scenarios is evident, which is a consequence of the small block size selected.

Shown in Fig. 4 are the averaged bit error rates for turbo error correction at $20 \mathrm{Mbit} / \mathrm{s}$ with 10 equi-power users. It can be seen that for low turbo iteration levels, there is no performance gain in the use of structured codes. As the number of iterations increases, however, an average improvement of half a decade is achieved at $\mathrm{SNR}=18 d B$. This indicates that system improvement does result from the use of structured mapping codes, which subsequently increase the free distance within the trellis decoder. Thus, with an increased number of iterations the receiver is coming closer to the correct path through the trellis.

\section{CONCLUSIONS AND Future WORK}

The use of non-binary turbo codes with a symbol based decoding algorithm has shown to provide a performance improvement for a low number of users. The use of truncated 
hyperbolic congruence codes for the symbol mapping has been shown to achieve the same performance level as random codes in a single user system, while having the benefit of simpler design. For high traffic systems, it was shown that structured codes achieve lower error rates for a high number of turbo iterations.

\section{REFERENCES}

[1] FCC, "New public safety applications and broadband internet access among uses envisioned by fcc authorization of ultra-wideband technology," Unofficial announcement of Commission action, February 2002.

[2] - "Revision of part 15 of the commission's rules regarding ultra-wideband transmission systems," Document 00-163, ET Docket No. 98-153, April 2002. [Online]. Available: www.fcc.gov/ Bureaus/Engineering_Technology/Orders/2002/fcc02048.pdf

[3] M. Z. Win and R. A. Scholtz, "Impulse radio: How it works," in IEEE Communications Letters, vol. 2, no. 2. IEEE, Feb 1998, pp. 36-38.

[4] K. Usuda, H. Zhang, and M. Nakagawa, "Pre-rake performance for pulse based uwb system in a standardized uwb short-range channel," in Wireless Communications and Networking Conference, vol. 2, March 2004, pp. 920-925.

[5] K. Popovski, T. A. Wysocki, and B. J. Wysocki, "Combined user multiplexing and data modulation through non-binary turbo codes for uwb," in to appear in WCNC'08, 2008.

[6] P. Robertson and T. Worz, "Bandwidth-efficient turbo trellis-coded modulation using punctured component codes," in IEEE Journal on Selected Areas in Communications, vol. 16, no. 2, February 1998, pp. 206-218.

[7] S. Lin and J. D. J. Costello, Error Control Coding, 2nd ed. Pearson Prentice Hall, New Jersey, 2004.

[8] J. Tan and G. L. Stuber, "A map equivalent sova for non-binary turbo codes," in IEEE International Conference on Communications, vol. 2, June 2000, pp. 602-606.

[9] C. Berrou, M. Jezequel, C. Douillard, and S. Kerouedan, "The advantages of non-binary turbo codes," in ITW2001, Cairns, Australia, September 2001.

[10] M. M. Pietrzyk, K. Popovski, T. A. Wysocki, B. J. Wysocki, and J. H. Weber, "Scarcely populated uwb-ir systems with interleaved codingmodulation on multipath fading channels," in The 2006 IEEE 2006 International Conference on Ultra-Wideband, September 2006, pp. 5560.

[11] A. Swami, B. Sadler, and J. Turner, "On the coexistence of ultrawideband and narrowband radio systems," in Military Communications Conference, Communications for Network-Centric Operations: Creating the Information Force, vol. 1, October 2001, pp. 16-19.

[12] A. Derode, A. Tourin, J. de Rosny, M. Tanter, S. Yon, and M. Fink, "Taking advantage of multiple scattering to communicate with timereversal antennas," Phys. Rev. Lett, vol. 90, no. 1, pp. 014 301-1 $014301-4,2003$.

[13] A. Saleh and R. Valenzuela, "A statistical model for indoor multipath propagation," IEEE Journal on Selected Areas in Communications, vol. 5, no. 2, pp. 128-137, February 1987.

[14] J. Liu and G. Tu, "Iterative decoding of non-binary turbo codes using symbol based sova algorithm," in International Conference on Communications, Circuits and Systems, vol. 2, June 2006, pp. 689-693.

[15] O. Moreno and S. V. Maric, "A new family of frequency-hop codes," IEEE Transactions on Communications, vol. 48, no. 8, pp. 1241 - 1244 August 2000.

[16] A. C. Reid, D. P. Taylor, and T. A. Gulliver, "Non-binary turbo codes," in IEEE International Symposium on Information Theory, 2002, p. 57.

[17] L. Cong, W. Xiaofu, and S. Songgeng, "On sova for nonbinary codes," in Fifth Asia-Pacific Conference on Communications and Fourth Optoelectronics and Communications Conference, vol. 1, October 1999, pp. $641-643$.

[18] M.-. G. D. Benedetto and G. Giancola, Understanding Ultra Wide Band - Radio Fundamentals. Prentice Hall, 2004. 